Check for updates

Cite this: RSC Adv., 2019, 9, 20829

\section{Waste brewed tea leaf derived cellulose nanofiber reinforced fully bio-based waterborne polyester nanocomposite as an environmentally benign material}

\begin{abstract}
Geeti Kaberi Dutta and Niranjan Karak (D) *
Bio-resources have carved a unique niche for the ever-increasing thrust of the global scientific community to impart green credentials to various research outputs along with the demands for advanced materials. In this milieu, the authors wish to fabricate a fully bio-based waterborne polyester nanocomposite as an advanced material using different bio-based reactants and cellulose nanofibers as the nanomaterial. Three different compositions of the nanocomposite were prepared at different loadings of cellulose nanofibers $(0.25,0.5$ and 1 weight\%) which were isolated from waste brewed green tea leaves. The structural attributes of the nanocomposites were evaluated by Fourier transform infrared spectroscopic, $\mathrm{X}$-ray diffraction, scanning electron microscopic and transmission electron microscopic studies. The nanocomposites were further cured with glycerol based epoxy and fatty acid based poly(amido amine) as the hardener to obtain the respective thermosets. The significant improvements in mechanical properties including tensile strength (13.71-22.33 MPa), elongation at break (128-290\%), toughness (15.65-45.18 $\mathrm{MJ} \mathrm{m}^{-3}$ ) and scratch hardness ( 8 to $>10 \mathrm{~kg}$ ) were observed for the thermosetting nanocomposites and the thermogravimetric analysis supports their high thermostability $\left(234-265^{\circ} \mathrm{C}\right)$. Further, the thermosetting nanocomposites were found to be highly biodegradable by Bacillus subtilis and Pseudomonas aeruginosa bacterial strains, hemocompatible with the erythrocytes present in RBCs and showed antioxidant properties. Thus, this nanocomposite could be used as a promising eco-friendly material for different related applications.
\end{abstract}

Received 21st April 2019 Accepted 24th June 2019 DOI: $10.1039 / c 9 r a 02973 g$ rsc.li/rsc-advances flexibility and overall low cost. The design of bio-based polyesters offers promise of sustainability on the growth of economically and ecologically attractive technology and hence in the recent times, much effort has been devoted for sustainable development of polyester by exploring renewable raw materials to replace petroleum based feedstocks. ${ }^{5-8}$ But, the synthetic procedures of the bio-based polyesters still depends on solvent, leading to evaporation of volatile organic compounds (VOCs) into the atmosphere causing environmental annoyance. So, Environmental Protection Agency and different environment quality monitoring bodies tried to use Green chemistry as a predestined tool to remove or decrease the amount of VOCs released to the atmosphere and promote growth of environment friendly sustainable chemical industries. Therefore, fully bio-based waterborne polyesters appears as an essential substitute to solvent borne system by decreasing the environmental pollution. Further, the biodegradability and biocompatibility are also essential factors of the bio-based polyesters in context to the ecological problems associated with their disposal and different applications in biomedical fields. ${ }^{9,10}$ However, the advanced applications of the sustainable polyesters are limited due to their poor mechanical strength
Advanced Polymer and Nanomaterial Laboratory, Department of Chemical Sciences, Tezpur University, Napaam, Tezpur, Assam, India, 784028.E-mail: karakniranjan@ gmail.com; Fax: +91-3712-267006; Tel: +91-3712-267009 
and thermal stability. In this context, the incorporation of suitable reinforcing nano-sized materials into the polyester matrices has come out to be an efficient way to modify and enhance their properties.

Among different nanomaterials, cellulose based nanomaterials have been of increasing interest due to their ubiquitous nature and abundance. Cellulose is a renewable and biodegradable plant polymer having low density with good mechanical properties and remarkable reinforcing capability which is due to the presence of $\beta-1,4$-D-glucose units linked by $\beta$ 1,4-glycosidic linkage and hydroxyl groups that enable cellulose to establish strong hydrogen bonds with the polar polymer matrices. They also possess environmental benefits due to which they have been utilized as potential nano-reinforcing filler to develop environmental friendly bio-based polymer nanocomposites or green nanocomposites for different biomedical and industrial applications. ${ }^{\mathbf{1 1 - 1 5}}$ However, in recent times, plants based cellulose has been exploited extensively for the production of nano-cellulosic material. ${ }^{16}$ Various sources of cellulose are wood, cotton, sisal, sugarcane bagasse, corncob, cassava bagasse, banana rachis, soy hulls etc., but instead of destroying fresh plant resources, it is preferable to use waste materials of plants for isolation of nano-cellulose and hence authors have used waste brewed green tea leaves for isolation of cellulosic nanofibers (CNF). There are numerous reports found in literature on cellulose based polymer nanocomposites with improved properties. Ansari et al. fabricated an isophthalic acid based unsaturated polyester/CNF bionanocomposite without using any coupling agents or surface modification; having improved modulus and strength of the unsaturated polyester along with improved ductility and toughness. ${ }^{17}$ Yao et al. fabricated a series of thermoplastic polyurethane/ microfibrillated cellulose nanocomposites via in situ polymerization which resulted in significantly improved mechanical properties and thermostability of the prepared nanocomposites. ${ }^{18}$ Goffin et al. used cellulose nanowhiskers to compatibilize binary polyester blends containing 50/50 (w/w) polycaprolactone (PCL) and polylactide (PLA), which further enhanced the mechanical properties of the blend and explained by the formation of continuous phase morphology at the interface. ${ }^{19} \mathrm{~A}$ viable route for the fabrication of epoxy resin and nanocellulose based nanocomposite was demonstrated by Yue et al. in which they impregnated a pre-percolated bacterial cellulose network with the bio-based epoxy resin followed by hot-pressing and curing, which further avoids fiber agglomeration and high viscosities. ${ }^{20}$ However, the reported cellulose polymer nanocomposites were not fabricated with fully biobased polymers and thus, as per our knowledge, no reports were found on fully bio-based waterborne polyester/CNF nanocomposites.

Therefore, the authors attempted to fabricate fully bio-based waterborne polyester (WBPE) nanocomposite via an environmentally benign route using waste brewed tea leaves derived $\mathrm{CNF}$ as the nano-reinforcing material. The mechanical, thermal, biodegradation, chemical resistance and radical scavenging properties of the fabricated polyester nanocomposites were further evaluated to judge their suitability as safe material for different potential applications. The authors also tried to generate the structure-property relationship of the fabricated polyester nanocomposite.

\section{Experimental}

\subsection{Materials}

Citric acid (Merck, India), glycerol (Merck, India), and dimer acid (Mn $\sim 570$, Sigma Aldrich, USA) were dried in a vacuum oven for about $4 \mathrm{~h}$ at $70{ }^{\circ} \mathrm{C}$ prior to use. Waste brewed green tea leaves were collected from local market. Oxalic acid (Rankem, India), potassium hydroxide (Rankem, India), para-toluene sulfonic acid (SRL, India), sodium sulfate anhydrous (Merck, India), ascorbic acid (Himedia, India), 1,1-diphenyl-2picrylhydrazyl (DPPH) (Himedia, India), glacial acetic acid and hydrogen peroxide (Merck, India), sodium hydroxide (Rankem, India), hydrochloric acid (Merck, India), dimethyl sulfoxide (Merck, India), ethanol (Merck, Germany), poly(amido amine) (Asian Paints, India, gift sample) and epichlorohydrin (Merck, India) were used as received. Bisphenol-A (Sisco Research Laboratories Pvt. Ltd., India) were recrystallized from toluene before use. Tetrahydrofuran (THF, SD fine Chem., India) was distilled before use. A glycerol based epoxy was prepared by following an earlier reported method. ${ }^{21}$ Briefly, required amount of glycerol, bisphenol-A and epichlorohydrin (1:2 mol ratio with respect to the total hydroxyl groups of the glycerol and bisphenol-A) are reacted along with dropwise addition of $5 \mathrm{~N}$ $\mathrm{NaOH}$ solution to the reaction mixture and the reaction was carried out at $110{ }^{\circ} \mathrm{C}$ for $4 \mathrm{~h}$. The organic phase was then separated out, washed thoroughly and dried under vacuum at $60{ }^{\circ} \mathrm{C}$ to obtain the desired epoxy resin. The bacterial strains of Pseudomonas aeruginosa and Bacillus subtilis were obtained from Department of Molecular Biology and Biotechnology, Tezpur University, India. All other chemicals used in this work were of reagent grade and used without any further purification.

\subsection{Isolation of CNF from waste brewed green tea leaves}

The isolation of CNF from waste brewed green tea leaves follows a series of chemical treatments. The tea leaves were filtered from the extracted tea beverage processed by pouring hot distilled water to the tea leaves at a tea/water ratio of $1 / 30 \mathrm{~g}$ $\mathrm{mL}^{-1}$ for 10 minutes. After the extraction and filtration, the tea leaves were grounded and bleached with $7 \% \mathrm{H}_{2} \mathrm{O}_{2}$ solution $(\mathrm{pH}$ adjusted to 5 with glacial acetic acid) for $2 \mathrm{~h}$ at $60{ }^{\circ} \mathrm{C}$ where the tea residue to solution ratio was maintained at $1 / 50 \mathrm{~g} \mathrm{~mL}^{-1}$. The bleached residue was then filtered through a micro filter paper and washed several times with distilled water until the $\mathrm{pH}$ was maintained to neutral. The residue was then treated with $17.5 \%$ sodium hydroxide for $3 \mathrm{~h}$ at $60{ }^{\circ} \mathrm{C}$ for complete delignification of the fibers. The alkaline treated solution was filtered and washed successively with distilled water to maintain a neutral $\mathrm{pH}$. Again the delignified cellulose residue was treated with 50\% aqueous glacial acetic acid solution for another $3 \mathrm{~h}$ at $60{ }^{\circ} \mathrm{C}$. The toxicity of the whole isolation process is reduced to some extent by using milder and less hazardous glacial acetic acid instead of toxic sulphuric acid and hydrochloric acid as reported in earlier 
methods. ${ }^{22,23}$ The acid treated fibers were then filtered and washed again with distilled water until neutral $\mathrm{pH}$. Afterwards, the fibers were treated with $50 \%$ aqueous DMSO solution for $2 \mathrm{~h}$ at $80{ }^{\circ} \mathrm{C}$. The $\mathrm{CNF}$ were collected by centrifugation and washed several times with distilled water. Then, the obtained CNF were mechanically dispersed in water to obtain a suspension of the nanofibers.

\subsection{Fabrication of WBPE/CNF nanocomposite}

The fabrication of the bio-based WBPE/CNF nanocomposite follows an in situ technique. The synthesis of WBPE was a two stage single pot process, in which required amount of dimer acid and glycerol were reacted in the first stage. The reaction was then carried out at $160{ }^{\circ} \mathrm{C}$ for $3 \mathrm{~h}$ in a three-neck round bottom flask under constant mechanical agitation and nitrogen atmosphere in the presence of 0.05 weight $\%$ of $p$-toluene sulphonic acid as the catalyst, until the acid value of the reaction mixture drops down to $5 \mathrm{mg}$ of $\mathrm{KOH}$ per $\mathrm{g} .{ }^{24}$ The obtained reaction mixture was then cooled down to $80^{\circ} \mathrm{C}$ with constant stirring and the required amount of citric acid was added along with the desired amount of CNF dispersed in minimum amount of EtOH to the above reaction mixture in the second stage. The reaction mixture was then allowed to react for another $1 \mathrm{~h}$ at $140{ }^{\circ} \mathrm{C}$ under constant mechanical agitation to get the desired polyester nanocomposite. By following the same protocol, three different resinous nanocomposites were fabricated using 0.25 , 0.5 and 1 weight $\%$ of CNF and coded them as PCNF 0.25, PCNF 0.5 and PCNF 1 respectively. The thermosets of these nanocomposites were obtained by amalgamating them with glycerol based epoxy (1:1 ratio w/w) and fatty acid based poly(amido amine) as the hardener ( 50 weight\% with respect to the epoxy) in the presence of minimum amount of THF at room temperature separately. This reaction mixture was then evenly coated on glass plates with dimensions of $75 \mathrm{~mm} \times 25 \mathrm{~mm} \times 1.3 \mathrm{~mm}$ for the measurement of tensile strength and scratch hardness; and on steel plates with dimensions of $150 \mathrm{~mm} \times 50 \mathrm{~mm} \times$ $1 \mathrm{~mm}$ for the measurement of impact resistance. The plates were then kept in a vacuum oven for 5 days to eliminate the entrapped solvent present in them and cured at $150{ }^{\circ} \mathrm{C}$ for a desired period of time. The optimizing curing time was calculated by checking the swelling value of the cured films. The cured films were then peeled out by immersing them in hot water and then dried. The thermosets were then coded as TPCNF 0.25, TPCNF 0.5 and TPCNF 1 respectively for the resinous nanocomposites PCNF 0.25, PCNF 0.5 and PCNF 1. WBPE in absence of CNF was also prepared by the reported method and the thermoset of the same was coded as WBPET and used for comparison purpose.

\subsection{Characterization}

Fourier transformed infrared (FTIR) spectra of the nanocomposites were recorded by a Nicolet (Impact-410, USA) infrared spectrometer using $\mathrm{KBr}$ pellets over the range of wavenumber from $4000-400 \mathrm{~cm}^{-1}$. The diffraction patterns of $\mathrm{CNF}$ and the thermosetting nanocomposites were studied by an X-ray diffractometer (XRD) (Bruker AXS, D8 FOCUS, Germany).
The crystallinity index of the CNF was calculated according to the empirical formula given below proposed by Segal, Creely, Martin, \& Conrad (1959). ${ }^{25}$

$$
\left.I=\left[I_{200}-I_{\mathrm{am}} / I_{200}\right)\right] \times 100
$$

where $I_{200}$ is the peak intensity corresponding to the (200) plane and $I_{\mathrm{am}}$ is the intensity of the valley between the peaks corresponding to the (200) and (110) planes. The UV spectra were recorded using an Evolution-300 UV-visible spectrophotometer (Thermo Fisher, USA) at room temperature $\left(25^{\circ} \mathrm{C}\right)$. The size and morphology of CNF and fabricated nanocomposites were studied by TEM analysis (JEOL, JEMCXII, Japan with operating voltage of $200 \mathrm{kV}$ ). The mechanical properties including tensile strength and elongation at break of all the thermosetting nanocomposites were measured as per the ASTM D 638 by using Universal Testing Machine (model WDW-10, Jinan, China), with a $500 \mathrm{~N}$ load cell and $10 \mathrm{~mm} \mathrm{~min}^{-1}$ crosshead speed. A scratch hardness tester (model number 705, Sheen Instrument Ltd, UK) with stylus accessory and a travel speed of $50 \mathrm{~mm} \mathrm{~s}^{-1}$ was used to measure the scratch hardness of the thermosets. An impact tester (S.C. Dey \& Co., India, with $1 \mathrm{~m}$ as the maximum height) was used to measure the impact strength of the thermosetting nanocomposites following the standard ASTM D4272 falling weight method. A weight of $850 \mathrm{~g}$ was allowed to fall on the thermosets coated on steel plates from minimum to maximum falling heights just before any damage and the energy per unit thickness corresponding to the maximum height was taken as the impact strength. The decomposition profiles of the thermosetting nanocomposites were thermogravimetrically scrutinized under inert atmosphere of pure nitrogen gas with a flow rate of $30 \mathrm{~mL} \mathrm{~min}{ }^{-1}$ and heating rate of $5{ }^{\circ} \mathrm{C} \mathrm{min}{ }^{-1}$ in a temperature range of $30-700{ }^{\circ} \mathrm{C}$ by a TGA-4000 (PerkinElmer, USA) instrument. The chemical resistance of the thermosetting nanocomposites were performed as per the standard ASTM D 543-95 method in different chemical media including acidic medium ( $\mathrm{pH}=4-5)$, basic medium ( $\mathrm{pH}=8-9), 15 \% \mathrm{NaCl}$ solution and tap water. A small piece of each thermosets was weighted and immersed in different aforesaid chemical media for a specified period of 15 days at room temperature and the weight loss of all the individual films were measured after the completion of the test. The swelling\% for the thermosets was calculated by measuring the difference in weight between the dried thermoset $\left(W_{\mathrm{d}}\right)$ and the equilibrated swollen thermoset $\left(W_{\mathrm{s}}\right)$ as follows:

$$
\text { Swelling } \%=\left[\left(W_{\mathrm{s}}-W_{\mathrm{d}}\right) / W_{\mathrm{d}}\right] \times 100
$$

\subsection{Hemolytic assay}

The preliminary understanding about the cytocompatibility of the fabricated nanocomposites and their respective thermosets was gained by performing hemolytic assay which is a test to investigate the lysis of red blood cells by the targeted samples. Goat's blood was used to perform this experiment which was collected in a heparinized tube containing $4 \%$ sodium citrate 
solution from a slaughter house of local market, Tezpur, Assam, India. The collected blood was then centrifuged at $3000 \mathrm{rpm}$ for 30 minute at $4{ }^{\circ} \mathrm{C}$ to separate the erythrocytes from plasma. The separated erythrocytes were then washed for three times with phosphate buffer saline (PBS) for complete removal of plasma and a $5 \%$ hematocrit was obtained by re-suspending the packed erythrocytes in PBS $(10 \mathrm{mM}$ at $\mathrm{pH}=7.4)$ solution. The dispersions of the resinous nanocomposites and their thermosets with different concentrations $\left(0.5,2,4\right.$, and $\left.6 \mathrm{mg} \mathrm{mL}^{-1}\right)$ were prepared in water. The resinous nanocomposites were waterborne and hence they got easily dispersible in water. However, the thermosets are cross-linked product which cannot be dispersed in liquid medium so easily. So for that, the thermosets were cut into very tiny pieces which were then kept in stirring condition in water. Then $100 \mu \mathrm{L}$ of each samples were taken in microfuge tubes (Eppendorf) containing $1900 \mathrm{~mL}$ of $5 \%$ hematocrit which were then incubated for $30 \mathrm{~min}$ at $37^{\circ} \mathrm{C}$. After incubation, the samples were kept in an ice bath for one minute to terminate the samples, which is further followed by centrifugation for 5 minutes at $3000 \mathrm{rpm}$. The concentration of the hemoglobin was then determined by taking the UVabsorbance of the samples at $540 \mathrm{~nm}$ as a measure of hemolysis and the whole experiment was performed thrice to report an average result.

\subsection{Radical scavenging assay}

To perform DPPH radical scavenging assay, $1 \mathrm{~mL}$ of $0.1 \mathrm{M} \mathrm{DPPH}$ solution which was prepared with $50 \%$ aqueous ethanol solution, was mixed with $1 \mathrm{~mL}$ of each nanocomposites dispersions of different concentrations (0.4-2.0 $\mathrm{mg} \mathrm{mL}^{-1}$ in distilled water). The reaction mixture was then vortexed and incubated under dark conditions at room temperature for 20 minutes. The absorbance of the reaction mixture was then measured at $517 \mathrm{~nm}$ and the scavenging activity was measured by using the following formula

$$
\text { Scavenging effect }(\%)=\left[1-\left(A_{\mathrm{s}-517} / A_{\mathrm{c}-517}\right)\right] \times 100
$$

where $A_{\mathrm{s}-517}$ is the absorbance of the samples and $A_{\mathrm{c}-517}$ is the absorbance of the control, i.e.; ascorbic acid.

To perform the hydroxyl radical scavenging assay, $1 \mathrm{~mL}$ of each nanocomposites dispersions with different concentrations (0.4-2.0 $\mathrm{mg} \mathrm{mL}^{-1}$ in distilled water) was mixed with $2.4 \mathrm{~mL}$ of $0.1 \mathrm{M}$ phosphate buffer ( $\mathrm{pH} 7.4$ ) and $0.6 \mathrm{~mL}$ of $0.4 \mathrm{M} \mathrm{H}_{2} \mathrm{O}_{2}$ solution. The reaction mixture was then vortexed and incubated for 10 minutes at room temperature. The absorbance was then measured at $230 \mathrm{~nm}$ and the scavenging ability for hydroxyl radicals was calculated by using the following formula

$$
\text { Scavenging effect }(\%)=\left[1-\left(A_{\mathrm{s}-230} / A_{\mathrm{c}-230}\right)\right] \times 100
$$

where $A_{\mathrm{s}-230}$ is the absorbance of samples and $A_{\mathrm{c}-230}$ is the absorbance of the control, i.e.; ascorbic acid.

\subsection{Biodegradation study}

The thermosetting nanocomposites were tested for biodegradation following McFarland method by using Pseudomonas aeruginosa (Gram negative) and Bacillus subtilis (Gram positive) bacterial strains. ${ }^{26}$ Different salts such as $2 \mathrm{~g}$ of $\mathrm{Na}_{2} \mathrm{HPO}_{4}, 4.75 \mathrm{~g}$ of $\mathrm{KH}_{2} \mathrm{PO}_{4}, 1.2 \mathrm{~g}$ of $\mathrm{MgSO}_{4} \cdot 7 \mathrm{H}_{2} \mathrm{O}, 2 \mathrm{~g}$ of $\left(\mathrm{NH}_{4}\right)_{2} \mathrm{SO}_{4}, 100 \mathrm{mg}$ of $\mathrm{MnSO}_{4} \cdot 5 \mathrm{H}_{2} \mathrm{O}, 0.5 \mathrm{mg}$ of $\mathrm{CaCl}_{2} \cdot 2 \mathrm{H}_{2} \mathrm{O}, 100 \mathrm{mg}$ of $\mathrm{CuSO}_{4} \cdot 7 \mathrm{H}_{2} \mathrm{O}$, $10 \mathrm{mg}$ of $\mathrm{H}_{3} \mathrm{BO}_{3} \cdot 5 \mathrm{H}_{2} \mathrm{O}, 70 \mathrm{mg}$ of $\mathrm{ZnSO}_{4} \cdot 7 \mathrm{H}_{2} \mathrm{O}, 10 \mathrm{mg}$ of $\mathrm{MoO}_{3}$, $1 \mathrm{mg}$ of $\mathrm{FeSO}_{4} \cdot 7 \mathrm{H}_{2} \mathrm{O}$ were added to $1 \mathrm{~L}$ of demineralised water to prepare a medium which was further sterilized by autoclaving it for 15 minutes under a pressure of $103.5 \mathrm{kPa}$ at $120{ }^{\circ} \mathrm{C}$. The bacterial strains were then cultured by using this sterilized medium inside an incubator for $48 \mathrm{~h}$ at $37^{\circ} \mathrm{C}$. An amount of 100 $\mu \mathrm{L}\left(10^{8}\right.$ microbes per $\left.\mathrm{mL}\right)$ of this cultured bacterial medium was then added to $10 \mathrm{~mL}$ of the sterilized medium taken in a conical flask. The thermosetting nanocomposites which have to be tested for biodegradation were then sterilized at first under UV light of wavelength $254 \mathrm{~nm}$ and then added to the above prepared medium containing bacterial strains followed by incubation at $37{ }^{\circ} \mathrm{C}$. The negative control was the above medium without any thermosets. The bacterial growths on the thermosets were scrutinized by measuring the increase in turbidity with time which was further measured by the absorbance value at $600 \mathrm{~nm}$ as optical density (OD) of the microorganism. The values reported were the average of three experimental results which were performed for a period of 4 weeks. The images of degraded surface of the thermosets were taken by JEOL JSM-6390LV Scanning electron microscope.

\section{Results and discussions}

\subsection{Isolation of $\mathrm{CNF}$}

The hot distilled water extracts the polyphenols from green tea leaves in the tea beverage. The treatment of the waste green tea leaves with extensive hydrogen peroxide resulted in bleaching of the remaining polyphenols and lignocellulosic materials present in the leaves. In this step, the molecules displaying chromophoric groups or phenolic compounds in the lignin breaks down and their by-products are removed by whitening the pulp. Again, the alkali treatment hydrolyzed the constituents like starch and hemicelluloses; and thus facilitates in removal of lignins and hemicelluloses. Finally, the acid treatment preferentially hydrolyzes the amorphous regions of the fibers, whereas the crystalline regions were resistant to the acid attack and hence remain as there were. ${ }^{27,28}$ This nanomaterial was chosen not only because of its bio-origin or obtained from wastes, but also because the polar groups $(-\mathrm{OH})$ present in them can strongly interact with WBPE matrix.

\subsection{Characterization of $\mathbf{C N F}$}

FTIR spectrum of the isolated CNF is displayed in Fig. 1a which supports the chemical linkages present in fibers. The broad absorption band in the region of $3000-3600 \mathrm{~cm}^{-1}$ can be attributed to the $-\mathrm{OH}$ groups present in the fibers. The absorption band at $2912 \mathrm{~cm}^{-1}$ indicates the stretching vibration of the aliphatic saturated $\mathrm{C}-\mathrm{H}$ present in cellulose and hemicellulose. The peak observed at $1640 \mathrm{~cm}^{-1}$ is due to the $\mathrm{O}-\mathrm{H}$ bending of the adsorbed water in the cellulose. ${ }^{29}$ Again, the characteristic peak at $1431 \mathrm{~cm}^{-1}$ represents the $\mathrm{CH}_{2}$ scissoring 

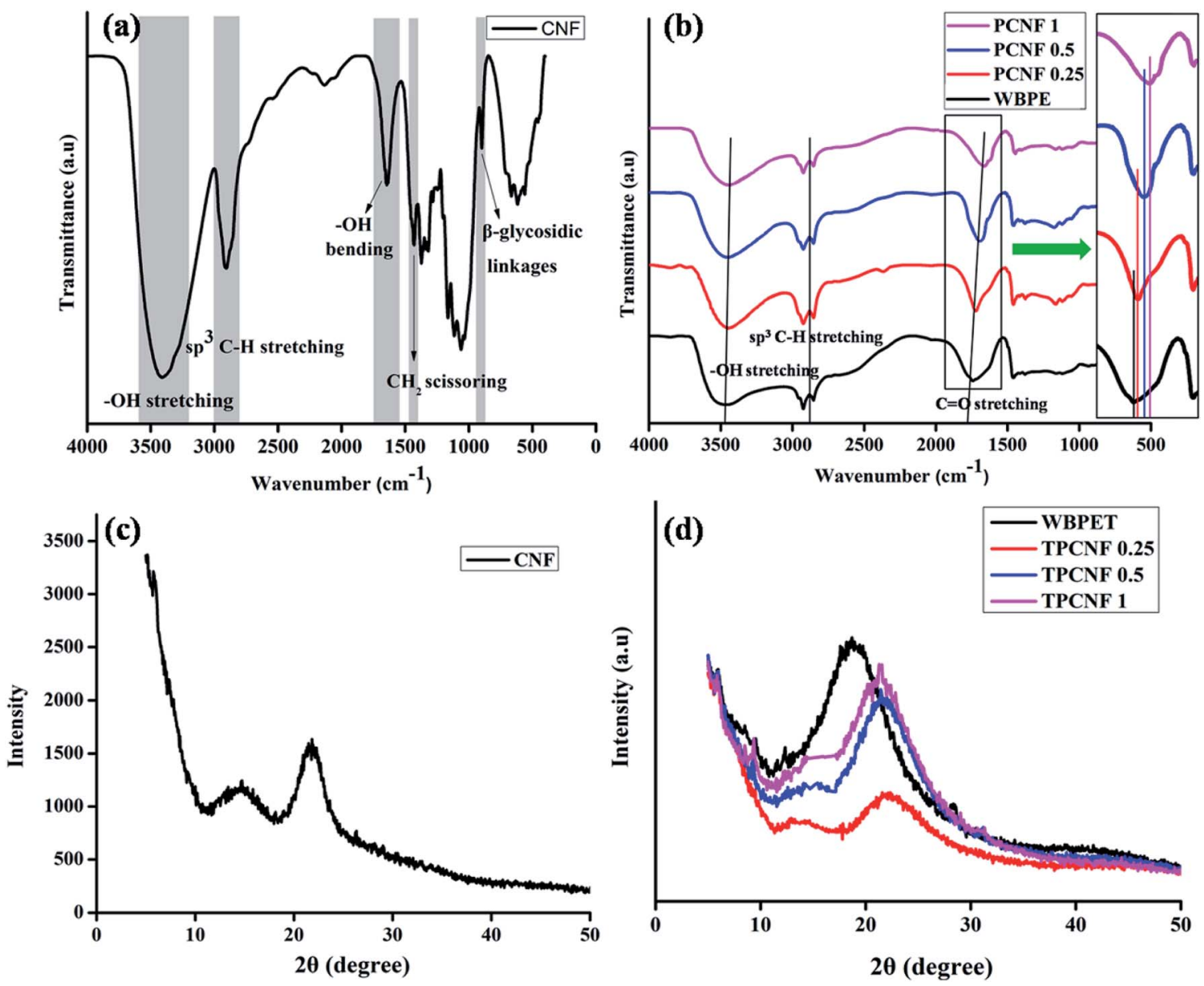

Fig. 1 (a) FTIR spectrum of CNF, (b) FTIR spectra of PCNF 0.25, PCNF 0.5, PCNF 1 and WBPE; (c) XRD pattern of CNF and (d) XRD patterns of TPCNF 0.25, TPCNF 0.5, TPCNF 1 and WBPET.

motion in cellulose and peak at $903 \mathrm{~cm}^{-1}$ represents the cellulosic $\beta$-glycosidic linkages. The remaining characteristic peaks are $1365 \mathrm{~cm}^{-1}$ (C-H bending), $1327 \mathrm{~cm}^{-1}$ (O-H in plane bending), $\sim 1157 \mathrm{~cm}^{-1}$ (C-C ring stretching band), $1104 \mathrm{~cm}^{-1}$ (C-O-C glycosidic ether band) and $1052 \mathrm{~cm}^{-1}$ (C-O-C pyranose ring stretching vibration). The absence of any peak at $1740 \mathrm{~cm}^{-1}$ and $1510 \mathrm{~cm}^{-1}$ further indicates the removal of most of the hemicelluloses and lignin from the fibers after the successive chemical treatments. ${ }^{30,31}$

To investigate the crystalline nature of the isolated CNF, XRD study of the same was performed and the diffraction pattern of the nanofiber sample is shown in Fig. 1c. Two diffraction peaks are observed in the figure at $2 \theta=14.7^{\circ}$ and $2 \theta=21.8^{\circ}$ which are typical for (110) and (200) characteristic planes of CNF. Further, the crystallinity index of the isolated CNF was calculated following eqn (1) and it was found to be about $44.8 \%$.

\subsection{Fabrication and characterization of the WBPE/CNF nanocomposite}

WBPE/CNF nanocomposite was fabricated by following an environmental benign, non-toxic, in situ technique in which no VOC was generated during the processing. Further, the interaction between CNF and WBPE matrix is expected to be very strong because of the favourable functionalities. In addition, the precursors used for fabrication of the polyester and nanomaterial are fully bio-based which is in accordance with one of the principles of green chemistry. In the first step of the polyester fabrication, a prepolymer melt was synthesized by reacting dimer acid and glycerol. The pre-polymer melt is nothing but oligomers which are low molecular weight resinous product that takes active part in the polymerization process after the addition of citric acid and required amount of CNF dispersed in EtOH; resulting in the formation of polyester resin as the final product. CNF were dispersed in EtOH to

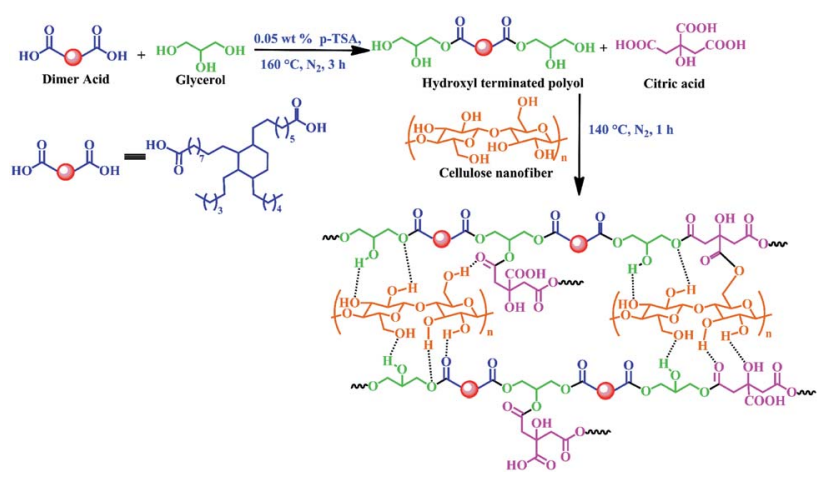

Scheme 1 Fabrication route of WBPE/CNF nanocomposite. 

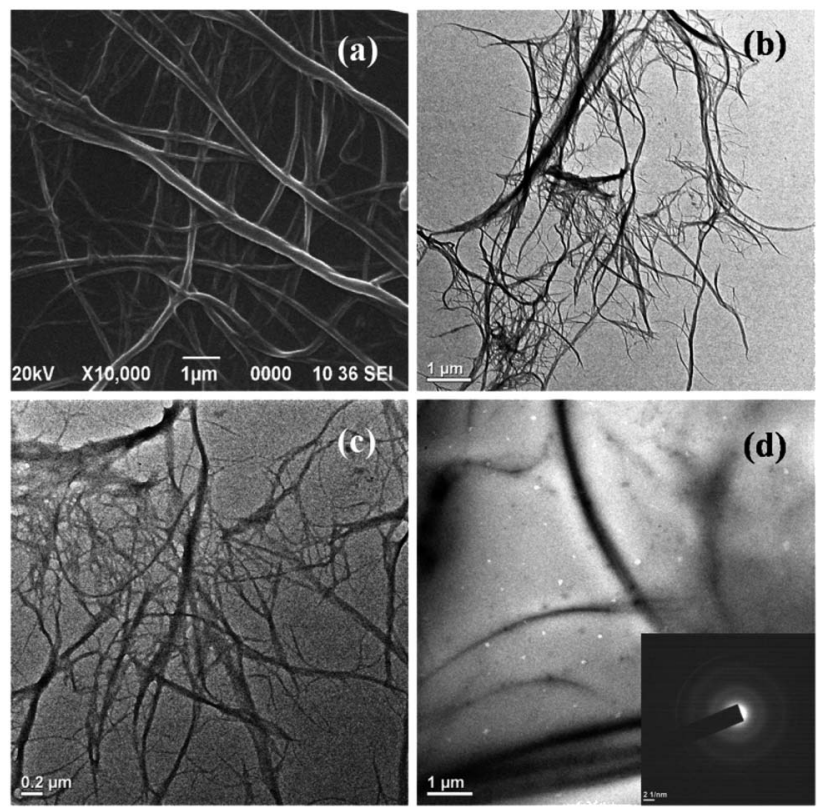

Fig. 2 (a) SEM image of CNF, (b and c) TEM images of CNF at different magnifications for morphological observation, (d) TEM image of PCNF 1 (with SAED pattern as inset).

form a uniform and stable dispersion due to the presence of strong interaction between their polar hydroxyl groups. As the viscosity of the pre-polymer is not high, so dispersion of CNF in that step is not a problem. The $-\mathrm{OH}$ functional groups of $\mathrm{CNF}$ further acquire an intrinsic tendency to interact covalently or non-covalently with WBPE as shown in Scheme 1. These types of interactions play a vital role towards the homogeneous dispersion of CNF in the polyester matrix and generates significant amount of interfacial area which is responsible for the improvement in the performances of the resultant nanocomposites.
The fabricated polyester nanocomposites are characterized by FTIR spectra (Fig. 1b) and XRD diffractograms (Fig. 1d). FTIR spectra support the different functional groups present in them. It is observed from Fig. 1b that the absorption bands of the nanocomposites are almost similar to the absorption bands of WBPE, except that the carbonyl stretching vibration is gradually shifted to lower frequencies $\left(1724 \mathrm{~cm}^{-1}\right)$ from $1743 \mathrm{~cm}^{-1}$. This shifting of the band positions indicates the interactions of the polyester carbonyl groups with the $-\mathrm{OH}$ groups of $\mathrm{CNF}$ through H-bonding or other polar-polar interactions. The peak around $3400 \mathrm{~cm}^{-1}$ in FTIR spectra of the nanocomposites is broadened compared to that of WBPE which indicates hydrogen bonding between -OH groups of WBPE and CNF as well as strong interactions in between them. Again, the absorption peaks at $2926 \mathrm{~cm}^{-1}$ and $2854 \mathrm{~cm}^{-1}$ indicates the asymmetric and symmetric stretching vibrations of aliphatic $\mathrm{C}-\mathrm{H}$ present in the resinous nanocomposites.

The crystallinity of the thermosets of the fabricated nanocomposites and the different interactions present in them were further confirmed from (Fig. 1d). The peak for WBPET at $18.6^{\circ}$ was shifted to higher angle $\left(21.7^{\circ}\right)$ after forming the nanocomposite which also coincides with the peak for CNF. The shifting of the peaks in the thermosetting nanocomposites compared with WBPET can be attributed to the formation of denser structure by strong interactions between the polyester matrix and the polar functionalities of CNF. Also with increasing the loading percentage of $\mathrm{CNF}$, the intensity of the peaks increases gradually which suggests that the crystallinity of the thermosetting nanocomposites increases and thus CNF is acting as a nucleating agent.

The morphology of the isolated CNF and the polyester nanocomposites were studied by TEM micrographs (Fig. 2b-d).

The micrographs revealed that the isolated CNF exhibited a traditional web-like network structure and occurred as long
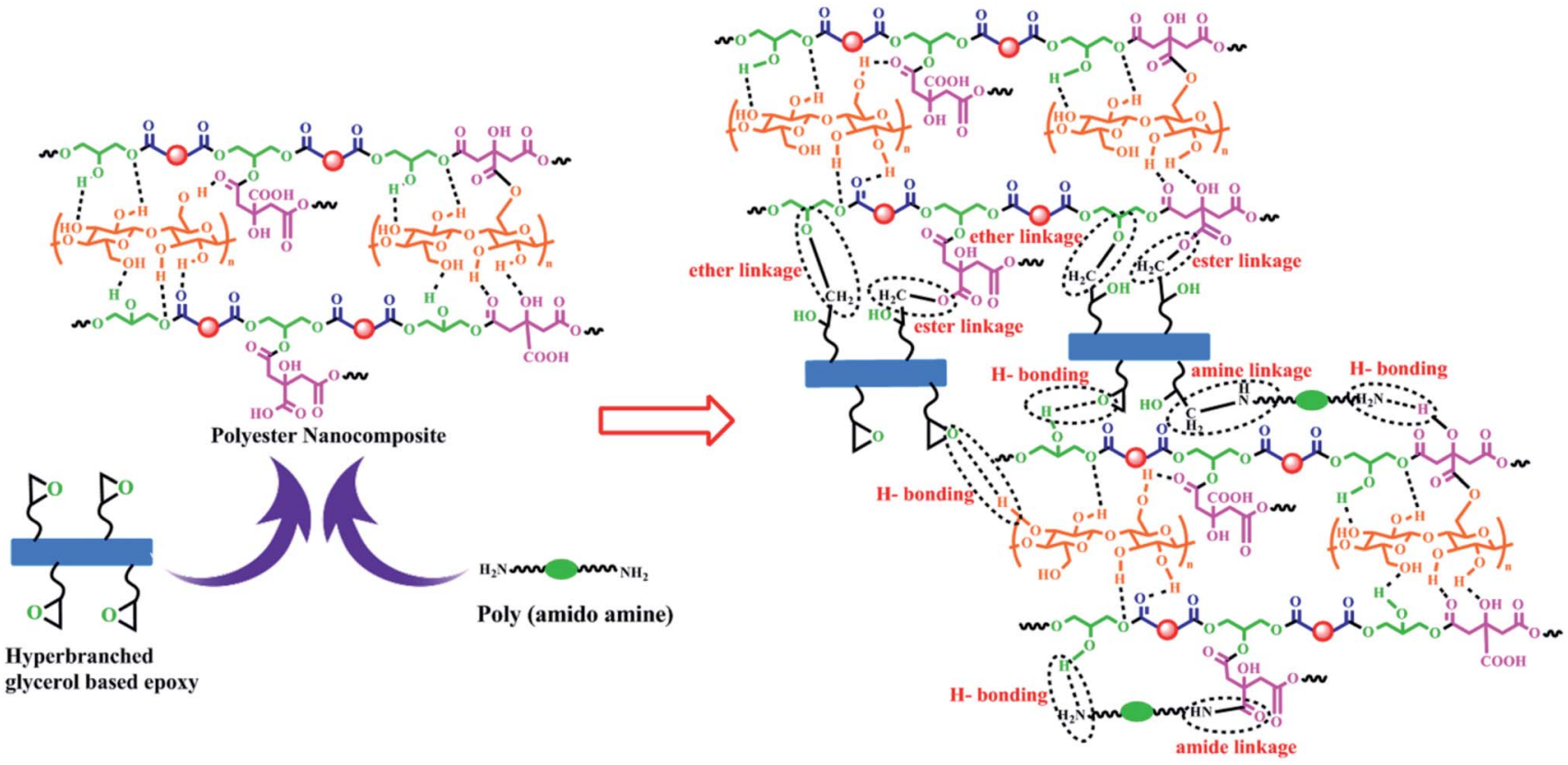

Scheme 2 Possible cross-linking reactions of WBPE/CNF nanocomposite with glycerol based epoxy and poly(amido amine) hardener. 
Table 1 Performance characteristics of WBPET and thermosetting WBPE/CNF

\begin{tabular}{|c|c|c|c|c|}
\hline Property & WBPET & TPCNF 0.25 & TPCNF 0.5 & TPCNF 1 \\
\hline Tensile strength (MPa) & $13.71 \pm 0.19$ & $16.68 \pm 0.8$ & $18.06 \pm 0.5$ & $22.33 \pm 1$ \\
\hline Elongation at break (\%) & $128 \pm 6$ & $155 \pm 5$ & $226 \pm 6$ & $290 \pm 3$ \\
\hline Scratch hardness (kg) & $8 \pm 0.2$ & $9 \pm 0.5$ & $10 \pm 0.3$ & $10 \pm 0.2$ \\
\hline Impact resistance $\left(\mathrm{kJ} \mathrm{m}^{-1}\right)$ & $20.50 \pm 0.3$ & $23.06 \pm 0.6$ & $23.24 \pm 0.5$ & $28.65 \pm 0.3$ \\
\hline Curing time at $160^{\circ} \mathrm{C}(\mathrm{min})$ & 180 & $330 \pm 5$ & $300 \pm 5$ & $280 \pm 10$ \\
\hline
\end{tabular}

entangled cellulosic threads. These results were also supported by SEM micrographs of CNF with lower magnification (Fig. 2a). The fibers observed were found to have diameter ranging from 20-200 $\mathrm{nm}$ and length of several micrometers though it was difficult to measure the total fibril length from the TEM images. Further, the TEM micrograph of the nanocomposite showed regular dispersibility of CNF within the polyester matrix without agglomeration. The well dispersion of $\mathrm{CNF}$ is due to the chemical interactions between the functional groups of the polyester matrix and the fibers. Also the selected area electron diffraction (SAED) pattern of the nanocomposite confirmed its overall amorphous nature.

\subsection{Curing study of the polyester nanocomposites}

The fabricated polyester nanocomposites were further crosslinked with glycerol based epoxy and fatty acid based poly(amido amine) as the hardener to get their respective thermosets and evaluated for different performance characteristics. The glycerol based epoxy was obtained from glycerol, bisphenol-A and epichlorohydrin which are reacted in the presence of $5 \mathrm{~N} \mathrm{NaOH}{ }^{21}$ Though bisphenol-A has hazardous impact on environment, but it is mainly used to enhance its performance characteristics and thermostability. Also, bisphenol-A can be easily degraded by different bacteria and thereby reducing its hazardous nature. ${ }^{32,33}$ Moreover, the use of bio-based reactants makes the overall epoxy much greener compared to the commercially available petrobased epoxy. The curing mechanism of the nanocomposites follows complex reactions due to the presence of different reactive functional groups in the system like $-\mathrm{OH},-\mathrm{COOH},-\mathrm{NH}_{2}$, $-\mathrm{C}=\mathrm{O}$ etc. The possible cross-linking reactions during curing of the fabricated nanocomposites are shown in Scheme 2. The free hydroxyl groups present in WBPE and CNF reacts with the oxirane rings of the glycerol based epoxy to form ether linkages in between them. The free-COOH groups of WBPE reacts with the free - $\mathrm{OH}$ groups of $\mathrm{CNF}$, oxirane rings of the epoxy and amine groups of the hardener to form new ester linkages and amide linkages respectively. The amine groups of the harderner can react with the oxirane rings to form amine linkages.

Further, the remaining $-\mathrm{OH},-\mathrm{NH}_{2}$, oxirane rings and carbonyl groups in the system form hydrogen bond among each other. The curing time of all the nanocomposites are given in Table 1. It was also observed that the rate of curing of the nanocomposites is higher than WBPE and increases with the increase in CNF loading. This can be attributed to the increasing number of polar -OH groups which forms strong interfacial interactions with WBPE matrix leading to even homogenization of all the components in the system and thus the rate of cross-linking reactions increases.

\subsection{Mechanical properties}

Different mechanical properties of polymer nanocomposites generally depends on numerous factors such as shape, size, rigidity, loading amount and uniform dispersion of the nanomaterial in the polymer matrix, chain entanglement, presence of different polar linkages, physico-chemical cross-linking, rigidity, hydrogen bonding etc. ${ }^{34}$ Different mechanical properties of the fabricated thermosetting nanocomposites are tabulated in Table 1 and their stress-strain curves are given in Fig. 3. The results of WBPET were also given for comparison purpose. The main motive of incorporating CNF into WBPE matrix is to address the poor mechanical properties of the bio-based polyester. All the fabricated thermosetting nanocomposites demonstrated good mechanical properties in which the effect of the nanomaterial was observed as the function of its loading. It is observed that the tensile strength, elongation at break and toughness of the thermosetting nanocomposites improved significantly over WBPET and follow an increasing trend with the increase in the weight percentage of CNF content.

The increasing tensile strength of the thermosets can be attributed to the increasing number of $-\mathrm{OH}$ groups with increasing weight percentage of $\mathrm{CNF}$, which forms more number of intra-molecular hydrogen bonding, ester linkages and amide linkages during cross-linking with the epoxy and

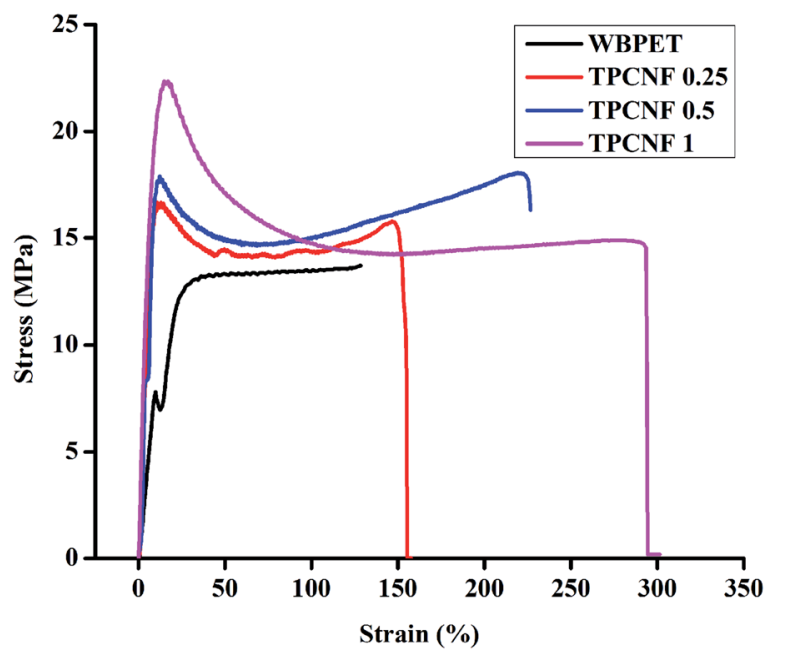

Fig. 3 Stress-strain curves of thermosetting WBPE/CNF nanocomposites. 
hardener. Thus, the overall free volume in the thermosetting nanocomposites matrix decreases leading to a rigid three dimensional geometry which makes them mechanically strong. The thermosetting nanocomposites exhibited improved tensile strength from 13.71 MPa to 22.33 MPa (62\% increment) with the highest loading of 1 weight\% of CNF which is far better result compared to the previously reported literature containing more amount (weight\%) of nanomaterial. ${ }^{35,36}$

The fabricated thermosets also followed an increasing pattern for the elongation at break (flexibility) with increase in CNF content. This can be attributed to the intricate architecture of the fabricated nanocomposite thermosets having extensive number of primary and secondary interactions which opens up during the time of elongation under the direction of applied force. The toughness, which is the combination of both tensile strength and flexibility of the thermosetting nanocomposites, calculated from the area under stress-strain curves, was found to be the highest for TPCNF 1. The improved toughness of TPCNF 1 was supported by enhanced scratch hardness values. All the thermosets with an average thickness maintained to $0.68 \mathrm{~mm}$ showed high impact resistance (limit of the instrument is $1 \mathrm{~m}$ ) following an increasing trend with increase in CNF content, which again demonstrates their high toughness character.

The surface morphology of the fractured pristine thermoset and fractured TPCNF 1 was also studied from the SEM images to

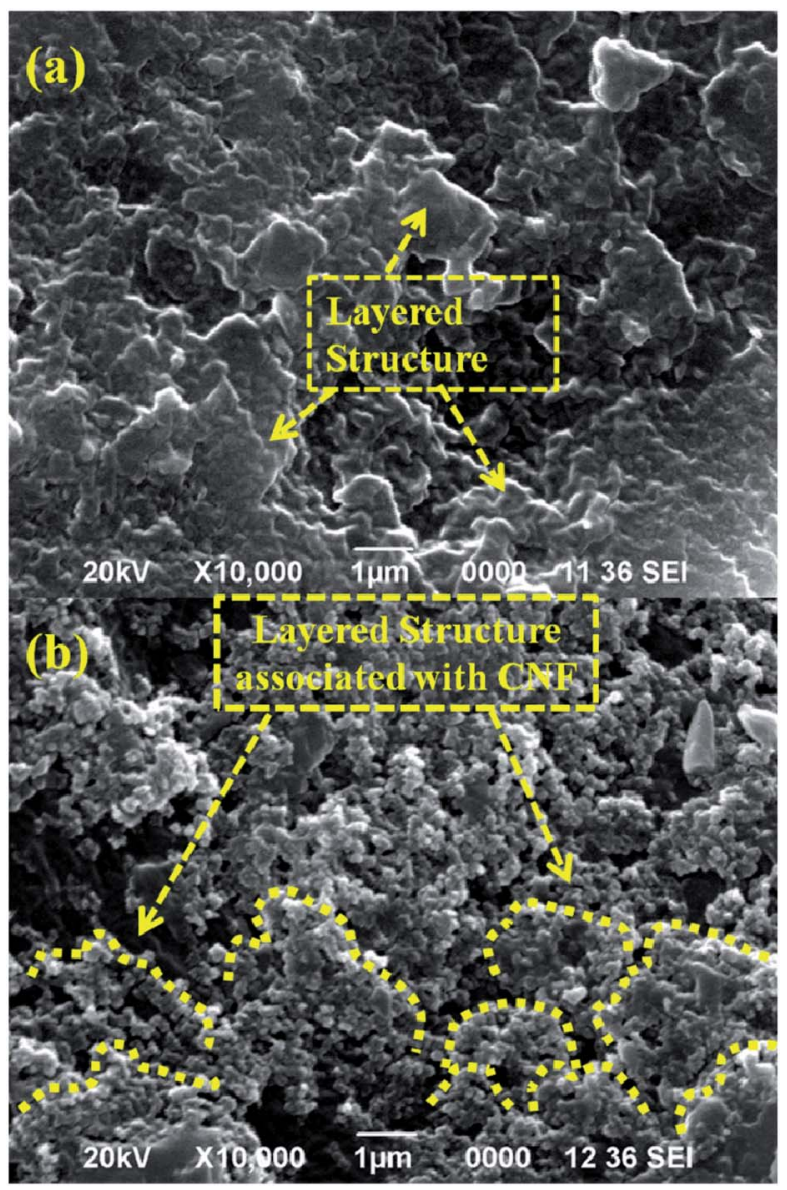

Fig. 4 SEM images of fractured surface of (a) WBPET and (b) TPCNF 1. get more detailed support on improvement of mechanical strength of the thermosetting nanocomposites after incorporation of CNF. The SEM image (Fig. 4a) of the fractured pristine thermoset revealed layered structure with low rough texture of the polyester matrix. On contrary, as observed in Fig. $4 \mathrm{~b}$, the nanocomposite has layered structure associated with CNF which formed an uneven texture with more micro-roughness on the fractured surface.

This observation suggested intercalation of CNF in between the polymeric chains by the formation of strong interfacial interactions in between them and thereby resulting in rough texture on the fractured surface of TPCNF 1. Further, such strong interactions between the polymeric chains and CNF allow smooth dissipation of applied force via synergistic effect resulting in high tensile strength and toughness.

\subsection{Thermal properties}

From the thermogravimetric analysis of the fabricated nanocomposite thermosets, it is observed that all the three compositions exhibited almost similar thermostability and follows
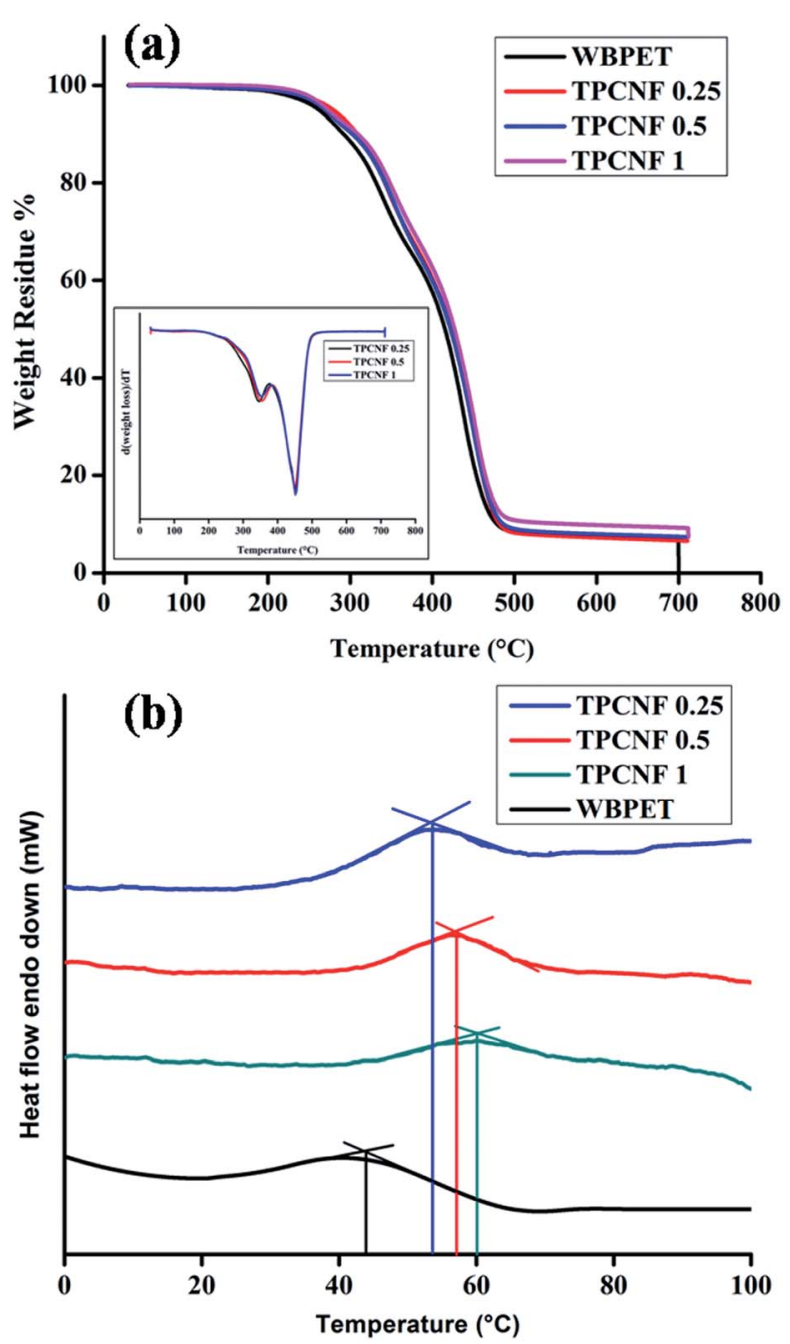

Fig. 5 (a) TGA thermograms with inset DTG curves of TPCNF 0.25 , TPCNF 0.5, TPCNF 1 and WBPET, (b) DSC curves of WBPE, TPCNF 0.25, TPCNF 0.5 and TPCNF 1. 
a two-step degradation pattern. The TGA thermograms and their respective DTG curves of the thermosets are shown in Fig. 5a. Also, the initial degradation temperature, peak temperature of first and second stages of degradation and weight residue percentage are tabulated in Table 2 . The first step degradation temperature which is in the range of 345$355{ }^{\circ} \mathrm{C}$ can be attributed to the thermos-labile ester linkages, ether linkages, amide linkages and aliphatic moieties, whereas the second step degradation temperature in the range of 450$458{ }^{\circ} \mathrm{C}$ can be attributed to the aromatic moieties and cycloaliphatic moieties. The aromatic moieties in the nanocomposite thermosets were contributed by bisphenol-A units present in the glycerol based epoxy whereas the cycloaliphatic moieties were present in CNF and in dimer acid unit of the polyesters. The cycloaliphatic moieties are predicted to get degraded at relatively high temperatures which are confirmed by the overlap in the second degradation steps. This is further supported by the observed slightly higher thermal stability of the thermosets with higher amount of CNF, even though it has equal amount of bisphenol-A based aromatic moieties. The weight residue left after degradation up to $700{ }^{\circ} \mathrm{C}$ were found to be $6.55 \%, 7.40 \%$ and $9.37 \%$ for TPCNF 0.25 , TPCNF 0.5 and TPCNF 1 respectively due to the formation of carbonaceous products. The weight residues were also found to increase with increase in CNF loading in WBPE.

The glass transition behaviour of the pristine polyester thermoset and the thermosetting nanocomposites were investigated by using differential scanning calorimetric (DSC) analysis and the DSC curves of the same are given in Fig. 5b. The pristine polyester thermoset exhibited glass transition temperatures $\left(T_{\mathrm{g}}\right)$ at around $44{ }^{\circ} \mathrm{C}$ and the thermosetting nanocomposites exhibited gradual increase in $T_{\mathrm{g}}$ from $53.6^{\circ} \mathrm{C}$ to $60{ }^{\circ} \mathrm{C}$ with increasing loading of $\mathrm{CNF}$. This increase in $T_{\mathrm{g}}$ indicates strong interactions such as hydrogen bonding between the polymeric chains of the nanocomposites and CNF which further reduces any segmental motion. However, no peaks are seen as the melting temperature in the DSC curves since the thermosets have cross-linked structure and hence no chances of melting.

\subsection{Chemical resistance}

The resistance of the fabricated thermosetting nanocomposites were studied under different chemical environments at room temperature for 15 days and their weight loss results are tabulated in Table 3. It is observed from the table that all the thermosets showed good resistance towards acidic medium of $\mathrm{pH}=$ 4-5, tap water and $10 \% \mathrm{NaCl}$ solution, but very poor resistance towards basic medium of $\mathrm{pH}=8-9$. It is also observed from the table that all the thermosetting nanocomposites showed better resistance than WBPET though the improvement is not that much noticeable. In general, the solutions of the different chemical media at first diffuse through the polyester matrix, occupy the voids present in them and disrupt the interfacial bonds via hydrolysis. Since the nanocomposites contains more number of free -OH groups compared to WBPE due to the presence of CNF, it forms highly dense structure during crosslinking with glycerol based epoxy and poly(amido amine) and hence, the diffusion of different chemical media through the nanocomposite matrix becomes difficult. However the free $-\mathrm{OH}$ groups also forms more number of hydrolysable ether linkages during cross-linking which are prone for disruption in basic and acidic media and hence the improvement of the chemical resistance of the thermosetting nanocomposites is not that much noticeable.

\subsection{Hemolytic assay}

For an advanced sustainable material to be considered as safe material, it is necessary to determine the health safety and potential hazard of the material during its life cycle. Thus, the

Table 2 Thermal degradation parameters of WBPET and thermosetting WBPE/CNF

\begin{tabular}{|c|c|c|c|c|}
\hline Parameter & WBPET & $\begin{array}{l}\text { TPCNF } \\
0.25\end{array}$ & $\begin{array}{l}\text { TPCNF } \\
0.5\end{array}$ & TPCNF 1 \\
\hline$T_{\text {on }}\left({ }^{\circ} \mathrm{C}\right)$ & 203 & 228 & 232 & 242 \\
\hline 2nd stage degradation peak temperature $\left({ }^{\circ} \mathrm{C}\right)$ & 432 & 450 & 449 & 452 \\
\hline Weight residue (\%) at $700{ }^{\circ} \mathrm{C}$ & 7.02 & 6.55 & 7.40 & 9.37 \\
\hline Glass transition temperature $\left({ }^{\circ} \mathrm{C}\right)$ & 44 & 53.6 & 57.1 & 60.4 \\
\hline
\end{tabular}

Table 3 Weight loss (\%) of the thermosetting nanocomposite in different chemical media

\begin{tabular}{lllll}
\hline & \multicolumn{2}{l}{ Weight loss $(\%)$} & & \\
\cline { 2 - 4 } Chemical medium & WBPET & TPCNF 0.25 & TPCNF 0.5 & TPCNF 1 \\
\hline Acidic medium (pH =4-5) & 0.422 & 0.412 & 0.406 & 0.416 \\
Basic medium (pH =8-9) & 2.011 & 2.006 & 2.001 & 0.0045 \\
$10 \%$ NaCl & 0.0059 & 0.0051 & 0.003 & 0.0039 \\
Tap water & 0.0019 & 0.0017 & 0.0005
\end{tabular}


safety of the fabricated sustainable nanocomposites was scrutinized by red blood cell (RBC) hemolytic protection assay as a representative biocompatibility test; in which hemocompatibility of the material was evaluated by detecting hemolyzation of erythrocytes. In this study, the material was brought in direct contact with the erythrocyte membrane and the extent of disruption of the membrane was evaluated as the direct measure of toxicity and further compared with hematocrit as the positive control and tween 20 as the negative control. The study revealed that all the compositions of the fabricated nanocomposite and their respective thermosets are well compatible with the erythrocytes compared to WBPE and WBPET; and among the three compositions; PCNF 1 and TPCNF 1 showed the highest compatibility as it contained the highest amount of biocompatible CNF (Fig. 6). The hemolyzation of the erythrocytes by the nanocomposites was determined from the absorbance value of the hemoglobin. The negative control, Tween 20 exhibited very high absorbance value indicating drastic rupture of the RBC membrane while the positive control, hematocrit exhibited very low absorbance value.

Thus, in vitro hemolytic assay confirmed the hemocompatibility of the fabricated resinous nanocomposite and their respective thermosets with mammalian RBC.

\subsection{Radical scavenging assay}

Diseases related to aging can be prevented and evaluated by determining the antioxidant status in biological systems. As antioxidation is essential for human physiological function, it is worthful to mention that oxidative stress is an important component of a material to be considered as safe, that can have an impact on the performance of medical devices. The antioxidant effectiveness of a biomaterial relies on its ability to stabilize the radicals by donating labile electrons or active hydrogen atoms and thereby partially oxidizing them. ${ }^{37}$ Thus, radical scavenging assays of the resinous nanocomposites and their respective thermosets against DPPH and hydroxyl radicals were determined; and it was observed that all the compositions of the nanocomposites showed profound antioxidant activities (Fig. 7). The results for the reaction of DPPH and PBS solution in the presence of $\mathrm{H}_{2} \mathrm{O}_{2}$ giving out hydroxyl radicals with different amount of the fabricated nanocomposites revealed a progressive decrease in the absorption intensity of DPPH at $517 \mathrm{~nm}$ and hydroxyl radicals at $230 \mathrm{~nm}$ with increasing concentration of the material.

The antioxidant activity of the nanocomposites thus increases with increasing concentration and also with
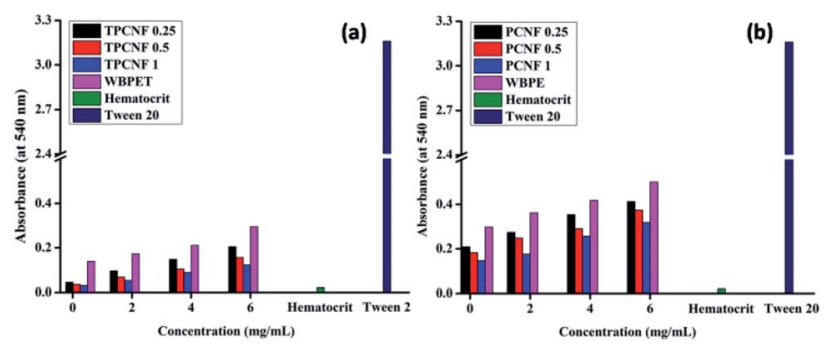

Fig. 6 Anti-hemolytic activity of (a) thermosetting nanocomposites and (b) resinous nanocomposite.
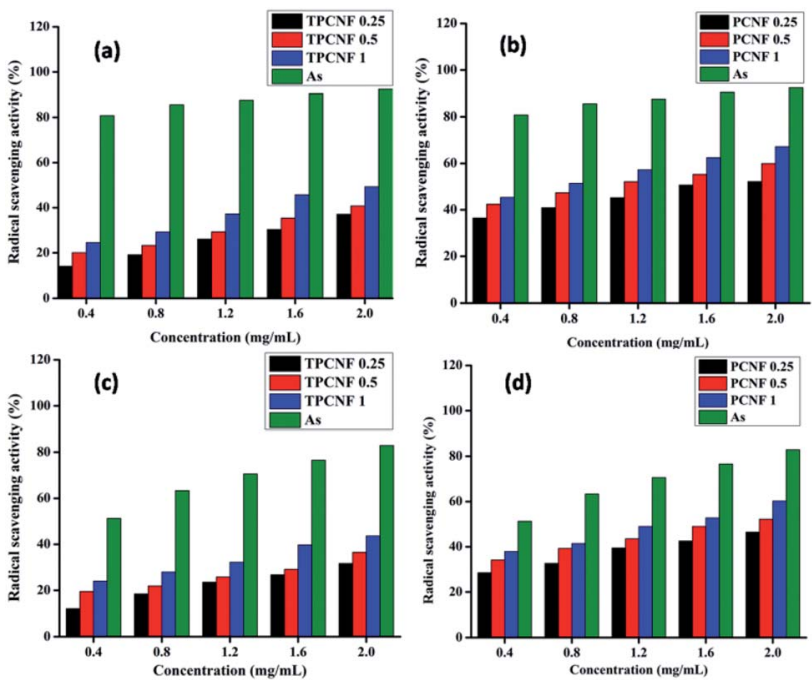

Fig. 7 DPPH radical scavenging activity of (a) thermosetting nanocomposites and (b) nanocomposite resins; hydroxyl radical scavenging activity of (c) thermosetting nanocomposites and (d) nanocomposite resins.

increasing CNF content and depends on the chemical structure of the material. The profound antioxidant activity of the nanocomposites can be attributed to the presence of large number of free hydroxyl groups in them. These free -OH groups can act as electron donor that reacts with the free radicals, stabilize them and terminate the radical chain reactions. From all the three compositions, PCNF 1 and TPCNF 1 exhibited the best radical scavenging activity whereas PCNF 0.25 and TPCNF 0.25 showed the least and this is attributed to the presence of greater number of $-\mathrm{OH}$ groups which is further due to the presence of greater amount of CNF.

\subsection{Biodegradation study}

The biodegradation study of the fabricated thermosetting nanocomposites were performed against Pseudomonas aeruginosa and Bacillus subtilis bacterial strains for a period of four weeks and the optical density (OD) values as well as the weight loss percentage of the degraded thermosets against exposure time was determined. It was found that the optical density values for the thermosetting nanocomposites were much higher than WBPET and it was gradually increasing with every week (Fig. 8a and b). Also, the weight loss percentages of the thermosetting nanocomposites were much higher compared with WBPET which confirms that the thermosets showed very high degradation (Fig. 8c). This can be attributed to the presence of more number of free -OH groups in the nanocomposites which forms additional hydrolysable ester, amide and ether linkages during cross-linking. The microorganisms are not able to take the polyesters directly into their cells for the biochemical processes because of their large size. Hence, they excrete extracellular enzymes which hydrolyze the labile linkages outside the cells causing surface erosion of the thermosets and forms more hydroxyl groups in the thermosetting nanocomposites. Thus, more the number of hydrolysable linkages in the thermosets, it becomes more easy for them to get degraded 

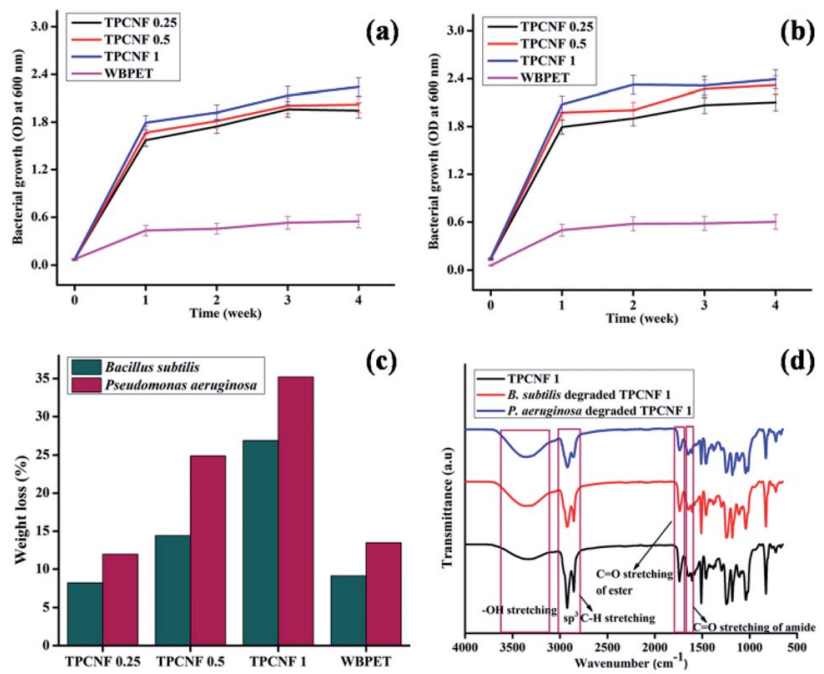

Fig. 8 Variation of optical density value against exposure time by (a) $B$. subtilis bacterial growth and (b) $P$. aeruginosa bacterial growth on the thermosetting nanocomposites; (c) weight loss percentages of thermosetting nanocomposites due to $B$. subtilis and $P$. aeruginosa bacterial growth; (d) FTIR spectra of TPCNF 1 before and after 4 weeks bacterial degradation.

leading to the formation of more number of hydroxyl groups. Later, it was observed that there is an increase in rate of degradation of the thermosetting nanocomposites with increase in CNF content which is again due to the presence of more number of $-\mathrm{OH}$ groups in them. It was also observed that the rate of degradation of the thermosetting nanocomposite were higher by the $P$. aeruginosa bacterial strain compared to the $B$. subtilis bacterial strain which can be seen from high weight loss percentages of the thermosets on exposure to the respective bacterial strains. This result is further confirmed from the FTIR spectra of the non-degraded TPCNF 1, B. subtilis degraded TPCNF 1 and $P$. aeruginos $a$ degraded TPCNF 1 given in Fig. 7d. It is observed from the spectra that the intensity of the
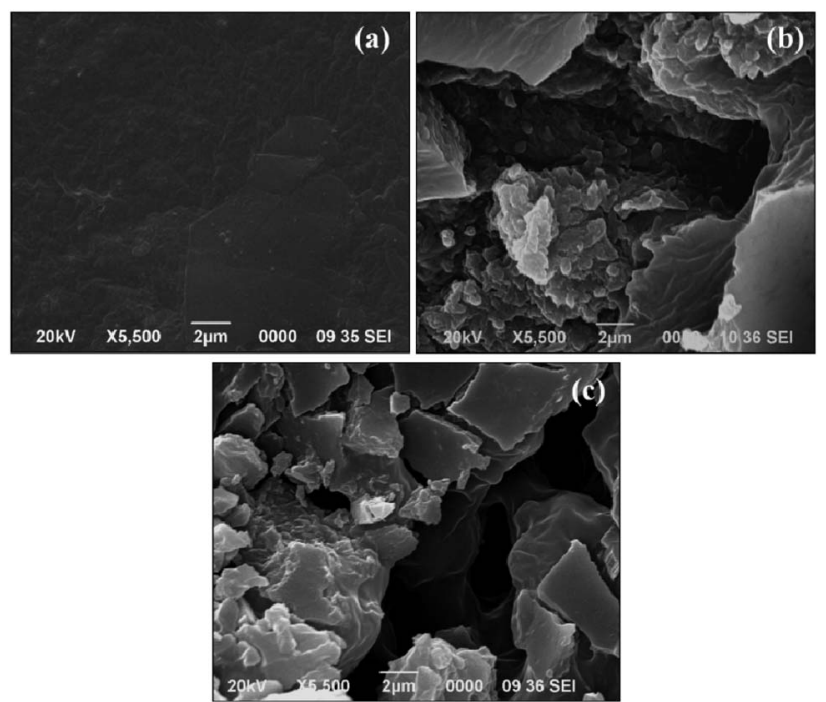

Fig. 9 SEM images of TPCNF 1 (a) control, (b) degraded by B. subtilis and (c) degraded by $P$. aeruginosa. carbonyl stretching of ester and amide linkages decreases compared to the non-degraded TPCNF 1 and the decrease is more pronounced in P. aeruginosa degraded TPCNF 1 than $B$. subtilis degraded TPCNF 1 . Also, the intensity of $-\mathrm{OH}$ stretching gradually increases for the degraded TPCNF 1 from the nondegraded TPCNF 1 and the increase is more pronounced in $P$. aeruginosa degraded TPCNF 1 compared to that of $B$. subtilis degraded TPCNF 1 . This is because, P. aeruginosa bacterial strain have high bio-surfactant activity and cell surface hydrophobicity (CSH) which leads to their faster bacterial colonization. Further, the SEM images (Fig. 9) of the degraded surface also confirmed biodegradation of the thermosetting nanocomposites.

\section{Conclusion}

From the study, it can be concluded that fully bio-based polyesters nanocomposites can be fabricated via an environmentally benign in situ technique. The cured thermosets of the nanocomposites are obtained after cross-linking them with glycerol based epoxy and poly(amido amine) which exhibited excellent mechanical, thermal and chemical resistance performances. The thermosets also showed high biodegradability which can be further tuned by varying amount of CNF. Also, the fabricated resinous nanocomposites and their respective thermosets are hemocompatible and have potent antioxidant activity. Thus the studied thermosetting waterborne polyester nanocomposite can find a decent position as a safe, sustainable and eco-friendly advanced material for different potential applications.

\section{Conflicts of interest}

There is no conflict of interest.

\section{Acknowledgements}

The authors express their gratitude to Council of Scientific and Industry Research (CSIR), New Delhi for financial assistance through granting the project No. 22(0759)/17/EMR-II. The authors would also like to acknowledge SAIF of NEHU, Shillong for TEM analysis and Sophisticated Analytical Instrumentation Centre (SAIC), Tezpur University for helping instrumental analyses.

\section{Notes and references}

1 R. Konwarh, N. Karak and M. Misra, Biotechnol. Adv., 2013, 31, 421-437.

2 A. Chakrabarty and Y. Teramoto, Polymers, 2018, 10, 517.

3 D. R. Paul and L. M. Robeson, Polymers, 2008, 49, 3187-3204.

4 L. Zhang, H. Zhang and J. Guo, Ind. Eng. Chem. Res., 2012, 51, 8434-8441.

5 Z. Wang, B. Kastern, K. Randazzo, A. Ugrinov, J. Butz, D. W. Seals, M. P. Sibi and Q. R. Chu, Green Chem., 2015, 17, 4720-4724.

6 Y. Xia and R. C. Larock, Green Chem., 2010, 12, 1893-1909. 
7 J. Dai, S. Ma, Y. Wu, L. Han, L. Zhang, J. Zhu and X. Liu, Green Chem., 2015, 17, 2383-2392.

8 C. Zhang, Y. Xia, R. Chen, S. Huh, P. A. Johnston and M. R. Kessler, Green Chem., 2013, 15, 1477-1484.

9 A. Asif and W. Shi, Eur. Polym. J., 2003, 39, 933-938.

10 G. K. Dutta and N. Karak, Prog. Org. Coat., 2019, 127, 419428.

11 C. Vilela, R. J. B. Pinto, A. R. P. Figueiredo, C. P. Neto, A. J. D. Silvestre and C. S. R. Freire, Development and applications of cellulose nanofibres based polymer nanocomposites, in Advanced Composite Materials: Properties and Applications, ed. E. Bafekrpour, 2017, pp. 1-65.

12 S. Kalia, A. Dufresne, B. M. Cherian, B. S. Kaith, L. Avérous, J. Njuguna and E. Nassiopoulos, Int. J. Polym. Sci., 2011, 135.

13 E. de Morais Teixeira, A. C. Corrêa, A. Manzoli, F. de Lima Leite, C. R. de Oliveira and L. H. C. Mattoso, Cellulose, 2010, 17, 595-606.

14 W. Chen, H. Yu, Y. Liu, Y. Hai, M. Zhang and P. Chen, Cellulose, 2011, 18, 433-442.

15 M. M. Haafiz, S. J. Eichhorn, A. Hassan and M. Jawaid, Carbohydr. Polym., 2013, 93, 628-634.

16 B. M. Cherian, A. L. Leão, S. F. de Souza, S. Thomas, L. A. Pothan and M. Kottaisamy, Carbohydr. Polym., 2010, 81, 720-725.

17 F. Ansari, M. Skrifvars and L. Berglund, Compos. Sci. Technol., 2015, 117, 298-306.

18 X. Yao, X. Qi, Y. He, D. Tan, F. Chen and Q. Fu, ACS Appl. Mater. Interfaces, 2014, 6, 2497-2507.

19 A. L. Goffin, Y. Habibi, J. M. Raquez and P. Dubois, ACS Appl. Mater. Interfaces, 2012, 4, 3364-3371.

20 L. Yue, F. Liu, S. Mekala, A. A. Patel, R. A. Gross and I. ManasZloczower, ACS Sustainable Chem. Eng., 2019, 7, 5986-5992.
21 S. Barua, G. Dutta and N. Karak, Chem. Eng. Sci., 2013, 95, 138-147.

22 A. Alemdar and M. Sain, Bioresour. Technol., 2008, 99, 16641671.

23 F. M. Pelissari, P. J. do Amaral Sobral and F. C. Menegalli, Cellulose, 2014, 21, 417-432.

24 G. K. Dutta and N. Karak, ACS Omega, 2018, 3, 16812-16822.

25 L. Segal, J. J. Creely, A. E. Martin and C. M. Conrad, Text. Res. J., 1959, 29, 786-794.

26 S. Dutta, N. Karak, J. P. Saikia and B. K. Konwar, Bioresour. Technol., 2009, 100, 6391-6397.

27 F. M. Pelissari, P. J. do Amaral Sobral and F. C. Menegalli, Cellulose, 2014, 21, 417-432.

28 S. Barua, G. Das, L. Aidew, A. K. Buragohain and N. Karak, RSC Adv., 2013, 3, 14997-15004.

29 M. Troedec, D. Sedan, C. Peyratout, J. Bonnet, A. Smith, R. Guinebretiere, V. Gloaguen and P. Krausz, Composites, Part A, 2008, 39, 514-522.

30 P. Garside and P. Wyeth, Stud. Conserv., 2003, 48, 269-275. 31 A. Kumar, Y. S. Negi, V. Choudhary and N. K. Bhardwaj, J. Mater. Phys. Chem., 2014, 2, 1-8.

32 J. H. Lobos, T. K. Leib and T. M. Su, Appl. Environ. Microbiol., 1992, 58, 1823-1831.

33 Y. H. Peng, Y. J. Chen, Y. J. Chang and Y. H. Shih, J. Hazard. Mater., 2015, 286, 285-290.

34 H. Kalita and N. Karak, Polym. Int., 2014, 63, 1295-1302.

35 K. Sakakibara, Y. Moriki, H. Yano and Y. Tsujii, ACS Appl. Mater. Interfaces, 2017, 9, 44079-44087.

36 M. Jonoobi, J. Harun, A. P. Mathew and K. Oksman, Compos. Sci. Technol., 2010, 70, 1742-1747.

37 E. N. Zarea, M. M. Lakouraj and M. Mohseni, Synth. Met., 2014, 187, 9-16. 